\title{
Comparative Study of Structural, Electrical, and Magnetic Behaviour of Ni-Cu-Zn Nanoferrites Sintered by Microwave and Conventional Techniques
}

\author{
Biju Thangjam ${ }^{1,2}$ and Ibetombi Soibam ${ }^{1}$ \\ ${ }^{1}$ Department of Physics, NIT Manipur, Imphal, Manipur 795004, India \\ ${ }^{2}$ Department of Physics, D. M. College of Science, Manipur University, Manipur 795001, India \\ Correspondence should be addressed to Biju Thangjam; bijuthdmc@gmail.com
}

Received 23 January 2017; Accepted 19 March 2017; Published 30 April 2017

Academic Editor: Christian Brosseau

Copyright (C) 2017 Biju Thangjam and Ibetombi Soibam. This is an open access article distributed under the Creative Commons Attribution License, which permits unrestricted use, distribution, and reproduction in any medium, provided the original work is properly cited.

\begin{abstract}
$\mathrm{Ni}_{0.8-x} \mathrm{Cu}_{x} \mathrm{Zn}_{0.2} \mathrm{Fe}_{2} \mathrm{O}_{4}$ spinel type ferrite nanoparticles have been synthesized by citrate precursor method. These nanoparticles were then given heat treatment using microwave and conventional sintering techniques. Various characterizations using $\mathrm{X}$-ray powder diffraction (XRD), scanning electron microscope (SEM), LCR meter, and $B$ - $H$ loop tracer were carried out on the sintered specimens. The XRD spectra of these ferrites confirmed the formation of spinel structure. The average crystallite size calculated using Scherrer's formula was found to be in the nanometer range, its value varying from $33 \mathrm{~nm}$ to $39 \mathrm{~nm}$. Microwave sintered samples exhibited superior electrical and magnetic behaviour over their conventionally sintered counterparts. Feasibility of low temperature synthesis and promising properties will render these ferrites suitable for multilayer chip inductor applications.
\end{abstract}

\section{Introduction}

Spinel nanoferrites have generated enormous interest in the scientific community throughout the world due to their unique properties. Nanocrystalline spinel ferrites have excellent electrical and magnetic properties like high resistivity, low eddy current losses, high saturation magnetization, high Curie temperature, and large permeability at high frequency which makes them suitable for a wide range of applications such as in information storage systems, magnetic cores, microwave absorbers, medical diagnostics, radio frequency circuits, high quality filters, rod antennas, transformer cores, magnetic resonance imaging (MRI), and drug-delivery technology [1-4]. A promising ferrite possessing the abovementioned characteristic features is the NiZn ferrite [5].

Considering the recent trend of technology, electronic devices are being processed in the form of microchips. Ferrites form a main component of such multilayer chip inductors (MLCIs) and are used with silver as the internal electrode. Since the melting point of silver is $961^{\circ} \mathrm{C}$, the sintering temperature of the ferrite should be below $961^{\circ} \mathrm{C}$ to prevent silver diffusion into the ferrite. This temperature is quite low to cofire with NiZn ferrites whose sintering temperature is $1250^{\circ} \mathrm{C}$. It has been reported that addition of $\mathrm{CuO}$ is capable of lowering the sintering temperature of NiZn ferrite with the conventional solid-state reaction method and coprecipitation method $[6,7]$. Hence, for the present investigation, Cu substituted NiZn ferrites have been synthesized using the citrate precursor method. This synthesis method has been reported as a versatile method for obtaining nanocrystalline ferrite particles. It allows homogeneous distribution of ions at the molecular level, good control of stoichiometry, small particle size formation, low temperature synthesis, and high efficiency. Besides, it is simple, inexpensive, and less timeconsuming $[3,8]$.

Lack of reports on a systematic investigation of the comparison of structural, electrical, and magnetic properties of $\mathrm{NiCuZn}$ ferrites sintered by microwave and conventional techniques prompted the present study. It has been reported that the microwave sintering technique tremendously 
reduces the sintering time as compared to the conventional one. This can be attributed to the fact that the microwave energy is delivered directly to the material through molecular interaction with the electromagnetic field resulting in volumetric heating, while in conventional sintering, heat is supplied to the sample through radiation, conduction, and convention and this needs a long sintering time which may lead to evaporation of certain constituents, thereby changing the desired stoichiometry [5]. Other prominent advantages of microwave heating over conventional heating are unique microstructure and properties, improved product yield, energy saving, reduction in manufacturing cost, and synthesis of new materials [9]. Therefore, in the present work, $\mathrm{Cu}$ substituted NiZn ferrite is prepared by citrate precursor method. Further, a comparative study on the structural, electrical, and magnetic properties of $\mathrm{NiCuZn}$ ferrites sintered by microwave and conventional sintering techniques is carried out and their possibilities for MLCI applications will be sought.

\section{Experimental Details}

2.1. Sample Preparation. $\mathrm{Ni}_{0.8-x} \mathrm{Cu}_{x} \mathrm{Zn}_{0.2} \mathrm{Fe}_{2} \mathrm{O}_{4}$ spinel ferrite nanoparticles with $0.0 \leq x \leq 0.5$ in steps of 0.1 were synthesized by the citrate precursor method using nickel nitrate $\mathrm{Ni}\left(\mathrm{NO}_{3}\right)_{2} \cdot 6 \mathrm{H}_{2} \mathrm{O}$ (Merck, India), copper nitrate $\mathrm{Cu}\left(\mathrm{NO}_{3}\right)_{2} \cdot 3 \mathrm{H}_{2} \mathrm{O}$ (Merck, India), zinc nitrate $\mathrm{Zn}\left(\mathrm{NO}_{3}\right)_{2} \cdot 6 \mathrm{H}_{2} \mathrm{O}$ (Merck, India), ferric nitrate $\mathrm{Fe}\left(\mathrm{NO}_{3}\right)_{3} \cdot 9 \mathrm{H}_{2} \mathrm{O}$ (Merck, India), and citric acid $\mathrm{C}_{6} \mathrm{H}_{8} \mathrm{O}_{7}$ (Merck, India). Stoichiometric amounts of nitrates and citric acid were dissolved separately in doubled distilled water. Metal nitrate solutions were mixed with citric acid solution in 1:1 molar ratio of nitrate to citric acid. The $\mathrm{pH}$ value of the solution was adjusted to 7 using ammonia solution. The resulting solution was heated at $100^{\circ} \mathrm{C}$ to form a viscous gel. Finally, the gel ignited in a self-propagating manner to form a fluffy loose ferrite powder. The as-burnt ferrite powders were ground using mortar and pestle. These powders were then mixed with $10 \%$ polyvinyl alcohol (PVA) as a binder and pressed to form pellets (10 $\mathrm{mm}$ diameter) and toroidal rings $(30 \mathrm{~mm}$ outer diameter, $10 \mathrm{~mm}$ inner diameter, and $4 \mathrm{~mm}$ thickness) by applying a pressure of 10 ton $\mathrm{cm}^{-2}$. These specimens were presintered at $600^{\circ} \mathrm{C}$ for $4 \mathrm{~h}$ in a programmable conventional furnace to expel the binder. The pellets and toroidal rings were then subjected to final sintering at $900^{\circ} \mathrm{C}$ ( $2 \mathrm{~h}$ for conventional sintering). Another set of samples was subjected to microwave sintering at a temperature of $900^{\circ} \mathrm{C}$ with a holding time of $30 \mathrm{~min}$ at a heating rate of $10^{\circ} \mathrm{C} / \mathrm{min}$. Finally, the samples were furnace-cooled. The pellets were coated with silver paste to provide electrical contact and the toroidal rings were wound with 70 turns of 30 SWG enamelled copper wire to form toroids.

Microwave sintering was carried out using a microwave furnace procured from VB Ceramic Consultants, Chennai, India. The microwave furnace uses a domestic microwave oven (VB Ceramic) with magnetrons operating at $2.45 \mathrm{GHz}$ frequency at a power output of $4.4 \mathrm{~kW}$. The temperature controller used was Eurotherm (Model 2416) microprocessor based PID programmer cum digital temperature indicator. The rate of heating and dwell with respect to time were preprogrammed and the entire operation was automatically controlled by the programmer. Temperature sensor output leads were directly connected to programmer as input. Raytek imported noncontact IR sensor was used to measure the temperature of sample directly. This sensor was fixed at the top of the furnace. $\mathrm{SiC}$ was selected as a microwave susceptor which reacted with the electromagnetic waves and created instant heat in the system. The specimen was placed in a crucible inside the cavity of the susceptor and was carefully placed in the furnace box. The sample centre and the centre of the sensor were aligned manually. The furnace was designed to work up to a maximum temperature of $1600^{\circ} \mathrm{C}$. Insulation zone was constructed with Zircar (USA) SALI grade high alumina insulation. Such type of insulation helps the furnace for the fast rate of heating and serves for severe thermal shock heating cycles. The main structure was made with high quality stainless steel and outer shell made with mild steel angles and sheets with neat powder coat finish.

2.2. Characterizations. The phase identification and structure analysis of the sintered samples were performed using Philips X'pert Pro X-ray diffractometer (microwave sintered samples) with $\mathrm{CuK} \alpha$ radiation $(\lambda=1.5406 \AA)$. The crystallite size of each composition was determined using Debye-Scherrer relation [10] and lattice parameters were calculated from the (311) diffraction peak. X-ray densities were also determined. The microstructures were examined by using FEI QUANTA scanning electron microscope (SEM). Dielectric measurements as a function of frequency in the range $20 \mathrm{~Hz}-2 \mathrm{MHz}$ were carried out at room temperature using a precision LCR meter E4980A. The electrical resistivity of the samples was determined. The real part of the dielectric constant was calculated using the formula

$$
\varepsilon^{\prime}=\frac{C d}{\varepsilon_{0} A},
$$

where $C$ is the capacitance of the pellet, $d$ is the thickness of the pellet, $A$ is the cross-sectional area of the flat surface of the pellet, and $\varepsilon_{o}$ is the permittivity of free space. Inductance $(L)$ of the sintered toroids was measured in the frequency range of $20 \mathrm{~Hz}-2 \mathrm{MHz}$ using a precision LCR meter E4980A. The measured inductance was used to calculate the initial permeability $\mu_{i}=L / L_{o}$, where $L$ is the measured inductance and $L_{o}$ is the air core inductance calculated using the relation

$$
L_{o}=\left(\frac{\mu_{o} N^{2} h}{2 \pi}\right) \ln \left(\frac{\mathrm{OD}}{\mathrm{ID}}\right),
$$

where $N$ is the number of turns of the coil $(N=70), h$ is the thickness, OD is the outer diameter, and ID is the inner diameter of the toroidal specimen. Soft magnetic properties were measured on toroidal core samples at room temperature. The coercivity $\left(H_{c}\right)$ and saturation magnetization $\left(M_{s}\right)$ were determined from $B-H$ hysteresis loops measured by $B-H$ loop tracer (Ferrites India) working on $50 \mathrm{~Hz}$ mains frequency. 
TABLE 1: Lattice parameter, X-ray density, experimental density, crystallite size, and resistivity values of $\mathrm{Ni}_{0.8-x} \mathrm{Cu}_{x} \mathrm{Zn}_{0.2} \mathrm{Fe}_{2} \mathrm{O}_{4}$ ferrites.

\begin{tabular}{|c|c|c|c|c|c|c|}
\hline \multirow[t]{2}{*}{ Samples " $x$ " } & \multirow{2}{*}{$\begin{array}{c}\text { Lattice parameter }(\AA) \\
\text { MW }\end{array}$} & \multirow{2}{*}{$\begin{array}{c}\text { X-ray density }\left(\mathrm{gm} / \mathrm{cm}^{3}\right) \\
\text { MW }\end{array}$} & \multirow{2}{*}{$\begin{array}{c}\text { Experimental density } \\
\left(\mathrm{gm} / \mathrm{cm}^{3}\right) \\
\mathrm{MW}\end{array}$} & \multirow{2}{*}{$\begin{array}{c}\text { Crystallite size } \\
\text { (nm) } \\
\text { MW }\end{array}$} & \multicolumn{2}{|c|}{ Resistivity $(\Omega$-cm $)$} \\
\hline & & & & & MW & Con \\
\hline 0.0 & 8.356 & 5.37 & 2.29 & 35.30 & $6.83 \times 10^{7}$ & $4.35 \times 10^{7}$ \\
\hline 0.1 & 8.362 & 5.37 & 2.58 & 36.84 & $4.44 \times 10^{7}$ & $6.28 \times 10^{7}$ \\
\hline 0.2 & 8.363 & 5.38 & 3.00 & 36.20 & $4.83 \times 10^{7}$ & $1.85 \times 10^{7}$ \\
\hline 0.3 & 8.369 & 5.38 & 3.34 & 34.26 & $3.34 \times 10^{7}$ & $2.32 \times 10^{7}$ \\
\hline 0.4 & 8.372 & 5.38 & 3.48 & 31.57 & $5.37 \times 10^{7}$ & $5.39 \times 10^{6}$ \\
\hline 0.5 & 8.375 & 5.39 & 3.30 & 32.65 & $2.13 \times 10^{7}$ & $4.14 \times 10^{6}$ \\
\hline
\end{tabular}

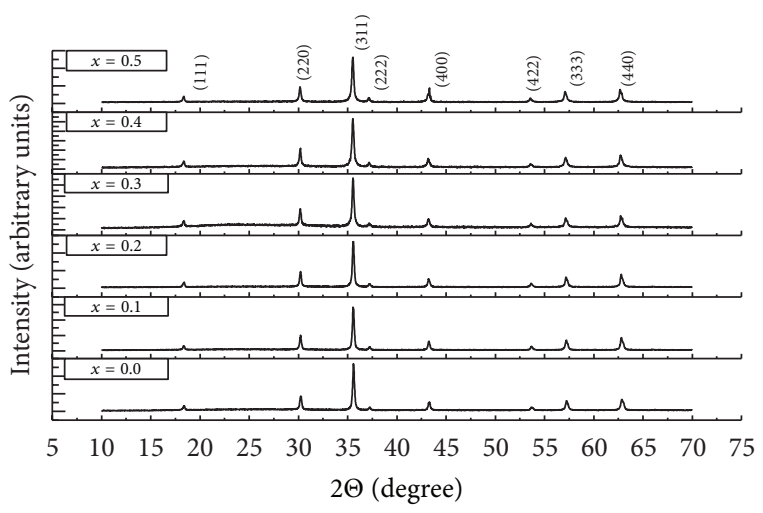

Figure 1: X-ray diffraction patterns of $\mathrm{Ni}_{0.8-x} \mathrm{Cu}_{x} \mathrm{Zn}_{0.2} \mathrm{Fe}_{2} \mathrm{O}_{4}$ (microwave sintering).

\section{Results and Discussion}

3.1. XRD and Structure Analysis. X-ray diffraction (XRD) patterns (Figure 1) of $\mathrm{Cu}$ substituted NiZn ferrites can be well indexed to JCPDS card number 01-071-3850, thereby establishing the formation of single phase cubic spinel structure. The patterns showed the absence of other impurity phases unlike the appearance of second phase of $\mathrm{CuO}$ or $\mathrm{CuFe}_{2} \mathrm{O}_{4}$ for ferrite composition of $x \geq 0.3$ prepared by coprecipitation method [11]. The XRD parameters like lattice constant, X-ray density, and crystallite size for the microwave sintered (MW) $\mathrm{Ni}_{0.8-x} \mathrm{Cu}_{x} \mathrm{Zn}_{0.2} \mathrm{Fe}_{2} \mathrm{O}_{4}$ ferrites are tabulated in Table 1. Lattice expansion occurs if the doping ion has larger radii than the displaced ion. In the present investigation, $\mathrm{Cu}^{2+}$ ions of larger ionic radius $0.72 \AA$ replace $\mathrm{Ni}^{2+}$ ions of smaller ionic radius $0.69 \AA$ and lattice constant is expected to increase. However, no significant increase is observed and this might be attributed to the fact that contribution to lattice constant does not solely depend on ionic radii but also on other interaction phenomena like the long range attractive coulomb force, bond length, and so forth which needs further investigation $[12,13]$. Besides, it has also been reported that the presence of copper ions in different ionic states in the $\mathrm{A}$ and $B$ sites is responsible for the nonlinear variation of lattice constant with increasing copper content [7]. An increasing trend in density (theoretical and experimental) was observed with the increase in $\mathrm{Cu}^{2+}$ ion concentration. This can be explained on the basis that atomic weight of $\mathrm{Cu}^{2+}(63.55 \mathrm{amu})$ is greater than that of $\mathrm{Ni}^{2+}(58.71 \mathrm{amu})$. The increase in experimental density with $\mathrm{Cu}^{2+}$ substitution may be attributed to the acceleration of cation interdiffusion due to $\mathrm{Cu}^{2+}$ ions and increase in reactivity of the fine ferrite grains which coalesce to form bigger grains resulting in pore reduction and volume shrinkage [14]. Also, the high atomic mobility of $\mathrm{Cu}^{2+}$ ions at relatively low temperature is an outstanding feature of $\mathrm{Cu}$ in increasing the density of the ferrite samples [15]. For a given value of $\mathrm{Cu}^{2+}$ concentration, the theoretical density is greater for the conventionally sintered samples (reported elsewhere) [16] than that of the microwave sintered ones. This could be due to longer sintering duration for the conventionally sintered samples. The same explanation will apply to the crystallite size as higher values of crystallite size are reported for the conventionally sintered samples [16] than the corresponding microwave sintered ones. The average crystallite sizes of all the samples are found to be in the range of $33 \mathrm{~nm}-39 \mathrm{~nm}$ clearly indicating that nanosized crystals can be effectively synthesized by the citrate precursor method.

3.2. Microstructural Characterization. The SEM micrographs for $\mathrm{Ni}_{0.8-x} \mathrm{Cu}_{x} \mathrm{Zn}_{0.2} \mathrm{Fe}_{2} \mathrm{O}_{4}$ nanoparticles are shown in Figure 2. Microstructural analysis allows determination of the average grain size of the samples (Table 2). The fine grained microstructural feature of these samples is effective for MLCIs with thinner internal ferrite layers. It is observed that grain size increases with increasing $\mathrm{Cu}^{2+}$ concentration up to $x=0.3$. During sintering, $\mathrm{Cu}^{2+}$ influences the microstructure by the formation of a liquid phase. The liquid phase induces higher atomic mobility of the $\mathrm{Cu}^{2+}$ ions and this facilitates the grain growth [17]. With further increase in $\mathrm{Cu}^{2+}$ content, the average grain size decreases. This may be owing to the fact that $\mathrm{Cu}^{2+}$ does not favour grain growth when present in excess amount. It has been reported that an intense microwave field concentrates around samples during microwave sintering resulting in ionization at the surface of ferrite particles. The kinetic energy of the ions at the grain boundaries increases due to the microwave field. This decreases the activation energy for a forward jump of the ions and increases the barrier height for a reverse jump. Hence, the forward diffusion of intergrain ions is enhanced, thereby accelerating the grain growth during sintering. As a result, grain size for the microwave sintered samples is found to be larger than the conventionally sintered ones [18]. 
TABLE 2: Grain size, initial permeability, $4 \pi M_{s}$, and coercivity values of $\mathrm{Ni}_{0.8-x} \mathrm{Cu}_{x} \mathrm{Zn}_{0.2} \mathrm{Fe}_{2} \mathrm{O}_{4}$ toroids.

\begin{tabular}{lcccccccc}
\hline \multirow{2}{*}{ Samples “ $x$ ” } & \multicolumn{2}{c}{ Grain size $(\mu \mathrm{m})$} & \multicolumn{2}{c}{ Initial permeability at $10 \mathrm{kHz}$} & \multicolumn{2}{c}{$4 \pi M_{s}$ (Gauss) } & \multicolumn{2}{c}{ Coercivity (Oersted) } \\
& MW & Con & MW & Con & MW & Con & MW & Con \\
\hline 0.0 & 0.16 & 0.12 & 4.14 & 3.26 & 144 & 156 & 0.59 \\
0.1 & 0.25 & 0.23 & 6.18 & 4.36 & 287 & 140 & 0.404 & 0.510 \\
0.2 & 0.40 & 0.32 & 24.63 & 7.34 & 1131 & 382 & 5.670 & 7.250 \\
0.3 & 0.47 & 0.45 & 31.54 & 9.78 & 1415 & 533 & 5.584 & 8.625 \\
0.4 & 0.37 & 0.32 & 19.02 & 7.55 & 1076 & 403 & 7.152 & 7.863 \\
0.5 & 0.44 & 0.42 & 24.61 & 8.54 & 1156 & 474 & 5.636 & 8.738 \\
\hline
\end{tabular}

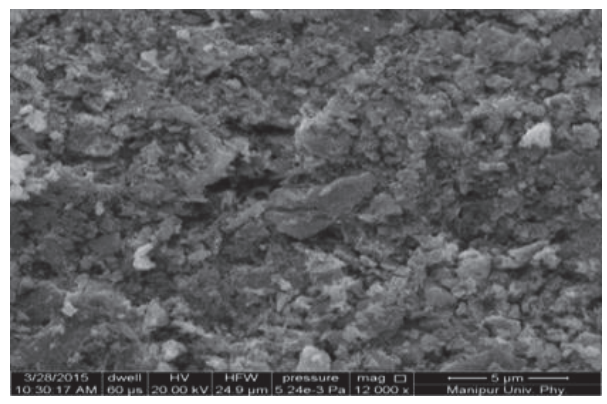

(a) $x=0.0$

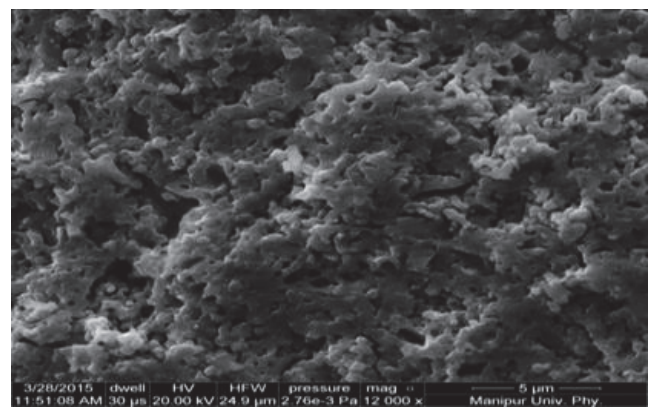

(c) $x=0.4$

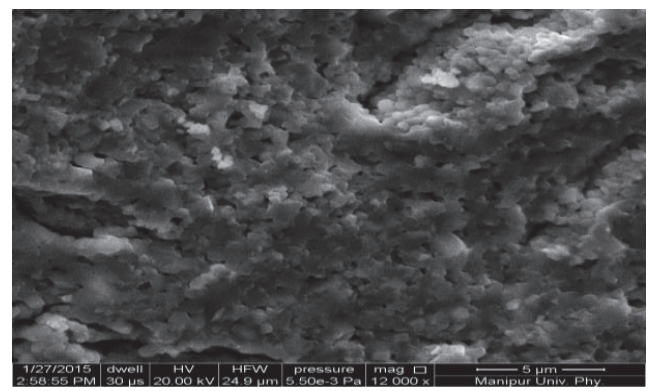

(e) $x=0.3$

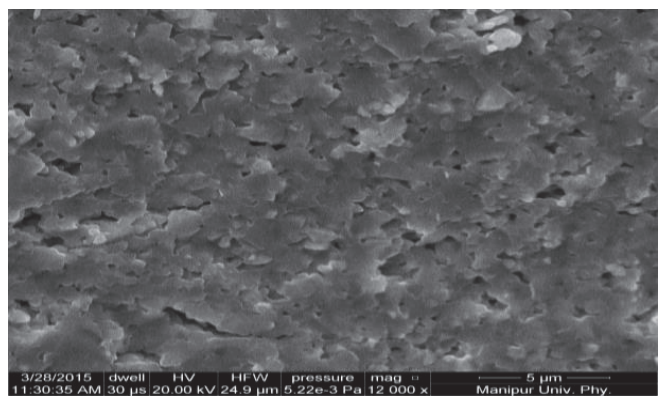

(b) $x=0.3$

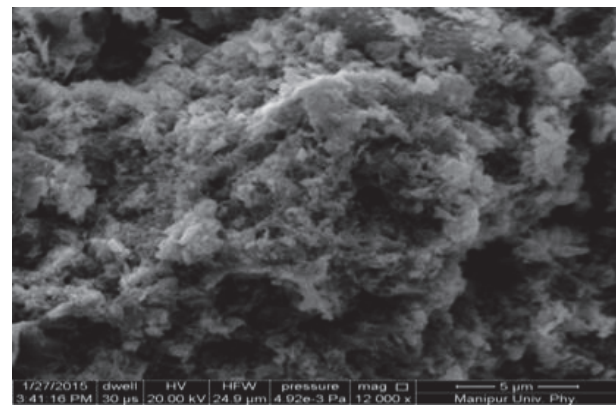

(d) $x=0.0$

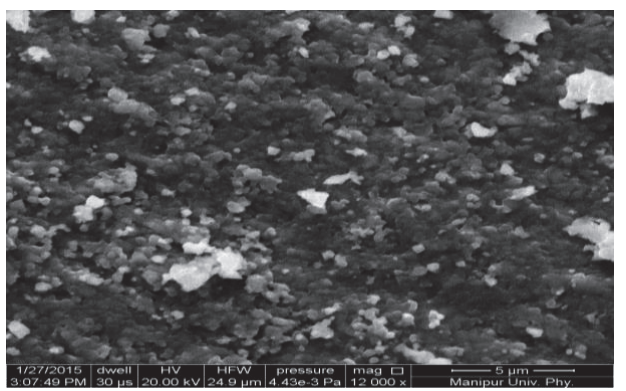

(f) $x=0.4$

Figure 2: SEM images ((a), (b), and (c)) of microwave sintered $\mathrm{Ni}_{0.8-x} \mathrm{Cu}_{x} \mathrm{Zn}_{0.2} \mathrm{Fe}_{2} \mathrm{O}_{4}$ ferrites and SEM images ((d), (e), and (f)) of conventional sintered $\mathrm{Ni}_{0.8-x} \mathrm{Cu}_{x} \mathrm{Zn}_{0.2} \mathrm{Fe}_{2} \mathrm{O}_{4}$ ferrites.

3.3. Dielectric Constant. The plots of variation of dielectric constant with frequency for both microwave sintered and conventionally sintered pellets are shown in Figures 3 and 4. It is observed that the decrease of dielectric constant at lower frequencies is rapid and becomes asymptotic to lower values at higher frequencies. This is a normal dielectric behaviour exhibited by spinel ferrites. Koop's two-layer model and Maxwell-Wagner polarization theory form the basis of the dielectric dispersion curve. According to Koop, relatively good conducting grains and insulating grain boundary layers of ferrite material can represent an inhomogeneous dielectric structure [19]. Verwey mechanism of electron hopping accounts for the electrical conduction in ferrites where electron hopping between $\mathrm{Fe}^{2+}$ and $\mathrm{Fe}^{3+}$ ions at $\mathrm{B}$ sites 


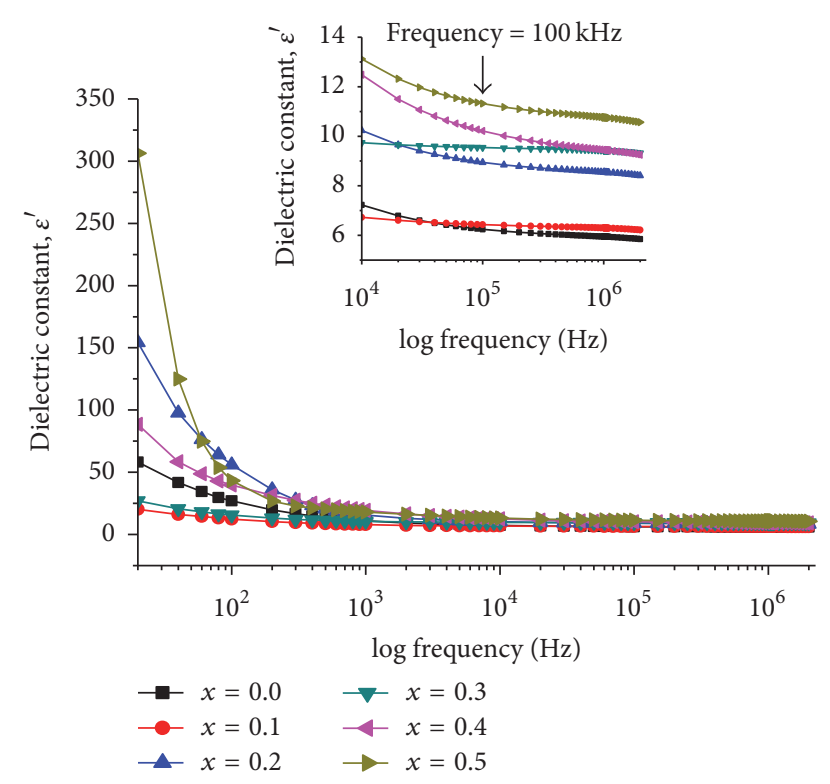

Figure 3: Frequency dependence of dielectric constant $\left(\varepsilon^{\prime}\right)$ for microwave sintered $\mathrm{Ni}_{0.8-x} \mathrm{Cu}_{x} \mathrm{Zn}_{0.2} \mathrm{Fe}_{2} \mathrm{O}_{4}$ ferrites.

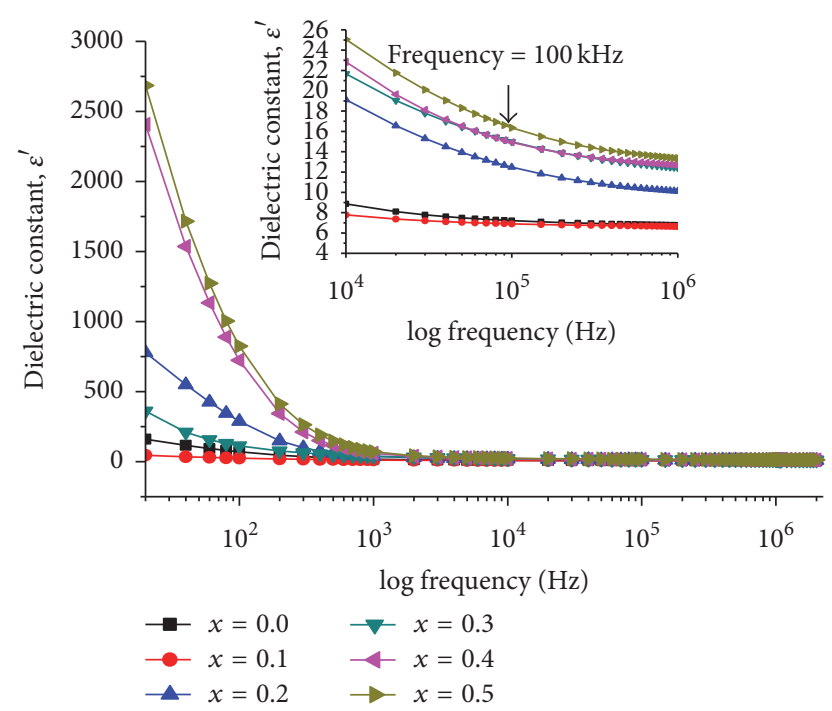

FIGURE 4: Frequency dependence of dielectric constant $\left(\varepsilon^{\prime}\right)$ for conventionally sintered $\mathrm{Ni}_{0.8-x} \mathrm{Cu}_{x} \mathrm{Zn}_{0.2} \mathrm{Fe}_{2} \mathrm{O}_{4}$ ferrites.

is responsible for the conduction [20]. During the process of electron hopping between $\mathrm{Fe}^{2+}$ and $\mathrm{Fe}^{3+}$ ions, localized electron accumulation takes place at the interface due to high resistance of the grain boundaries giving rise to space charge polarization. This results in a high value of dielectric constant at low frequencies. With further increase of frequency, the grains come into play, thereby reducing the value of dielectric constant rapidly. At still higher frequencies, the hopping of electrons cannot follow the frequency of the alternating field which leads to a decrease in contribution of space charge polarization. Hence, the dielectric constant decreases at high frequencies $[21,22]$.

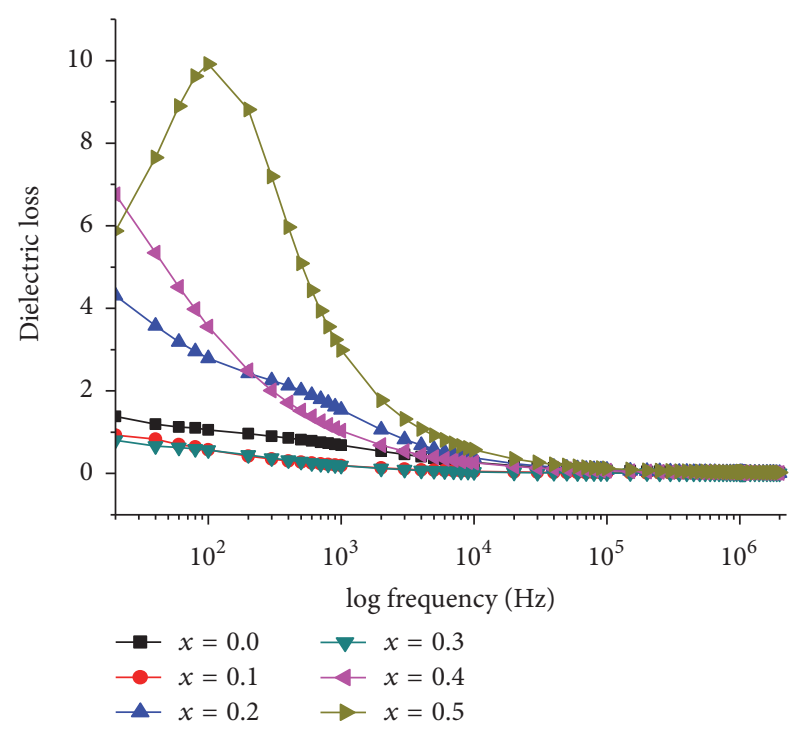

FIGURE 5: Variation of dielectric loss with frequency for microwave sintered $\mathrm{Ni}_{0.8-x} \mathrm{Cu}_{x} \mathrm{Zn}_{0.2} \mathrm{Fe}_{2} \mathrm{O}_{4}$ ferrites.

Various other factors affecting polarization are structural homogeneity, density, grain size, and porosity of ferrite material. Addition of $\mathrm{Cu}^{2+}$ in NiZn ferrite causes a decrease in structural homogeneity of the sample which may account for the increase in polarization [14]. The hole exchange between $\mathrm{Cu}^{2+}$ and $\mathrm{Cu}^{1+}$ ions also plays a vital role in the conduction and polarization mechanism. The addition of $\mathrm{Cu}$ may enhance the concentration of $\mathrm{Fe}^{2+} / \mathrm{Fe}^{3+}$ pairs in the $\mathrm{B}$ site, thereby resulting in an increase of dielectric constant $[23,24]$. The increase in density may also be a major factor contributing to the increase in polarization with $\mathrm{Cu}^{2+}$ incorporation. This is so because the increase in density decreases the porosity which will result in a higher number of polarizing species per unit volume [14]. This may account for the higher values of dielectric constant for the conventionally sintered samples than the corresponding microwave sintered samples.

The value of dielectric constant of these samples is low as compared to that of pure NiZn ferrites synthesized by the conventional solid-state process. Low sintering temperature of the samples must have resulted in lowering the possibility of ions to exist in different oxidation states. This in turn reduces the probability of hopping of charge carriers. Hence, a low value of dielectric constant is observed [14].

The variations of dissipation factor with frequency at room temperature for all the samples are shown in Figures 5 and 6. The dielectric loss decreased with increasing frequency, which is a normal behaviour of ferrites. This can be attributed to the fact that, in the low frequency region, which corresponds to a high resistivity (due to grain boundary), more energy is necessary for electron transfer between $\mathrm{Fe}^{2+}$ and $\mathrm{Fe}^{3+}$ ions. Hence, the loss is high. The high frequency region corresponds to a low resistivity (due to the grain), so a small amount of energy is necessary for electron hopping between $\mathrm{Fe}^{2+}$ and $\mathrm{Fe}^{3+}$ ions at the octahedral site. Hence, 


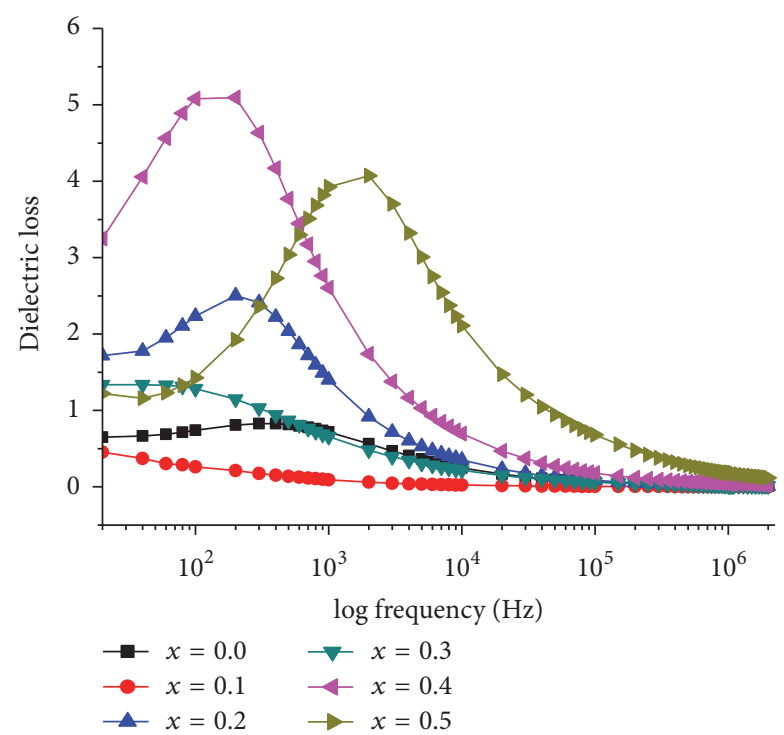

FIGURE 6: Variation of dielectric loss with frequency for conventionally sintered $\mathrm{Ni}_{0.8-x} \mathrm{Cu}_{x} \mathrm{Zn}_{0.2} \mathrm{Fe}_{2} \mathrm{O}_{4}$ ferrites.

the dielectric loss is low in this region. A peak at lower frequency region may be due to the existence of resonance between frequency of the externally applied electric field and the hopping frequency of the charge carriers. The domain wall resonance may also be a contributing factor [25].

Microwave sintered samples exhibited high loss factor values at lower frequencies, while the conventionally sintered samples possess relatively lower values. This can be explained by the fact that microwaves interact with high loss materials due to polarized species in the specimen. Exposure of highly absorbing species such as ferrites would result in the formation of polarized species within them [5].

3.4. DC Resistivity. The DC electrical resistivity is an important parameter of low temperature sintered ferrite for MLCI application. The variations of DC resistivity versus $\mathrm{Cu}^{2+}$ content for microwave (MW) and conventionally sintered (Con) pellets are shown in Table 1. Thakur and Singh [22] have reported that the variation of resistivity with $\mathrm{Cu}^{2+}$ content can be attributed to the grain size of the samples. Samples having small grains possess a higher number of grain boundaries. These boundaries are regions of mismatch between energy states of adjacent grains and hence act as a barrier to the flow of electrons. Additional advantage of small grain size is that it aids in reducing $\mathrm{Fe}^{2+}$ ions as oxygen moves faster in smaller grains and hence retains the iron in the $\mathrm{Fe}^{3+}$ state. This decreases the probability of electron hopping in smaller grains resulting in higher resistivity. However, resistivity may also be influenced by other factors like hopping of charge carriers, lattice site distribution, and so forth. The resistivity of the samples under study does not exactly follow the abovementioned trend, which needs further investigation.

3.5. Initial Permeability. The plots of the real part of initial permeability, $\mu_{i}^{\prime}$, versus frequency for all the toroids prepared

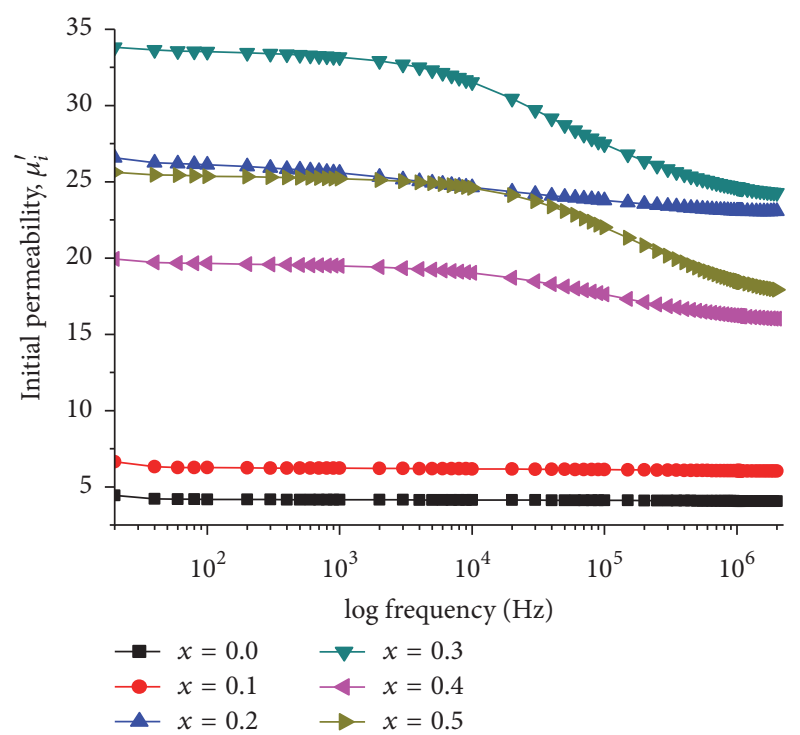

FIgURE 7: Frequency dependence of initial permeability $\left(\mu_{i}^{\prime}\right)$ of microwave sintered $\mathrm{Ni}_{0.8-x} \mathrm{Cu}_{x} \mathrm{Zn}_{0.2} \mathrm{Fe}_{2} \mathrm{O}_{4}$ ferrites.

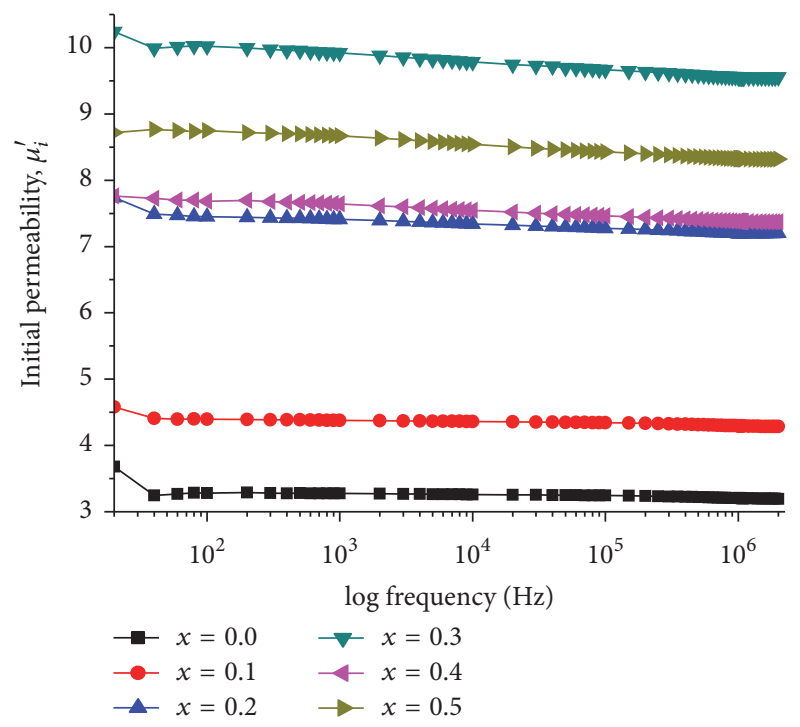

FIGURE 8: Frequency dependence of initial permeability $\left(\mu_{i}^{\prime}\right)$ of conventionally sintered $\mathrm{Ni}_{0.8-x} \mathrm{Cu}_{x} \mathrm{Zn}_{0.2} \mathrm{Fe}_{2} \mathrm{O}_{4}$ ferrites.

by microwave and conventional sintering techniques are shown in Figures 7 and 8 . It is observed that $\mu_{i}^{\prime}$ remains almost constant up to a frequency of $10 \mathrm{kHz}$ and thereafter it shows a gradual decline for the compositions with $\mathrm{Cu}^{2+}$ content of $0.2,0.3,0.4$, and 0.5 , while for the samples with $\mathrm{Cu}^{2+}$ content of 0.0 and 0.1 , the values of $\mu_{i}^{\prime}$ remain almost unchanged over the entire frequency range studied. It is reported that $\mu_{i}^{\prime}$ versus frequency curve is characterized by a critical frequency beyond which $\mu_{i}^{\prime}$ decreases rapidly from its static value. Initially, when there was great disparity between the drive frequency and the precision frequency, the value of $\mu_{i}^{\prime}$ remained static. The decrease in $\mu_{i}^{\prime}$ at a particular frequency is due to the onset of ferrimagnetic resonance. 


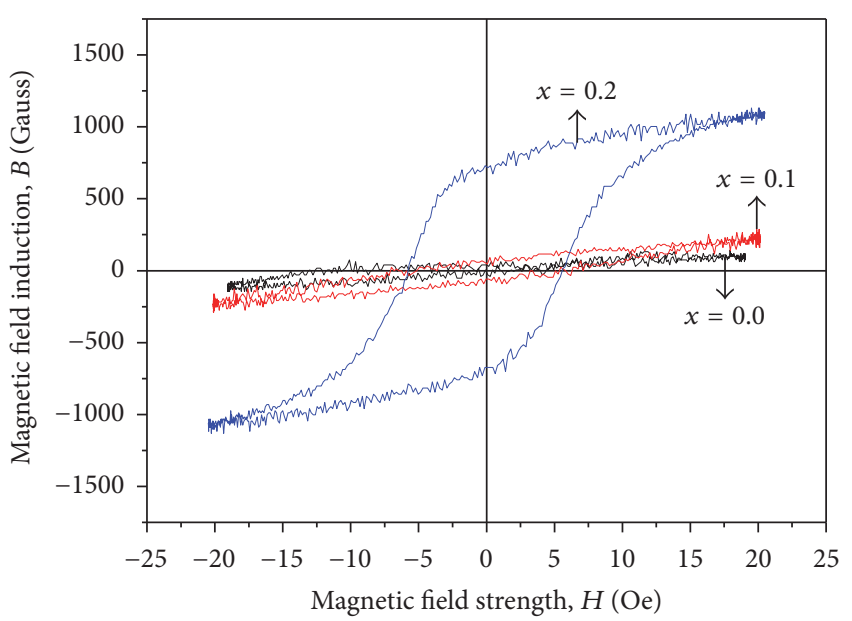

(a)

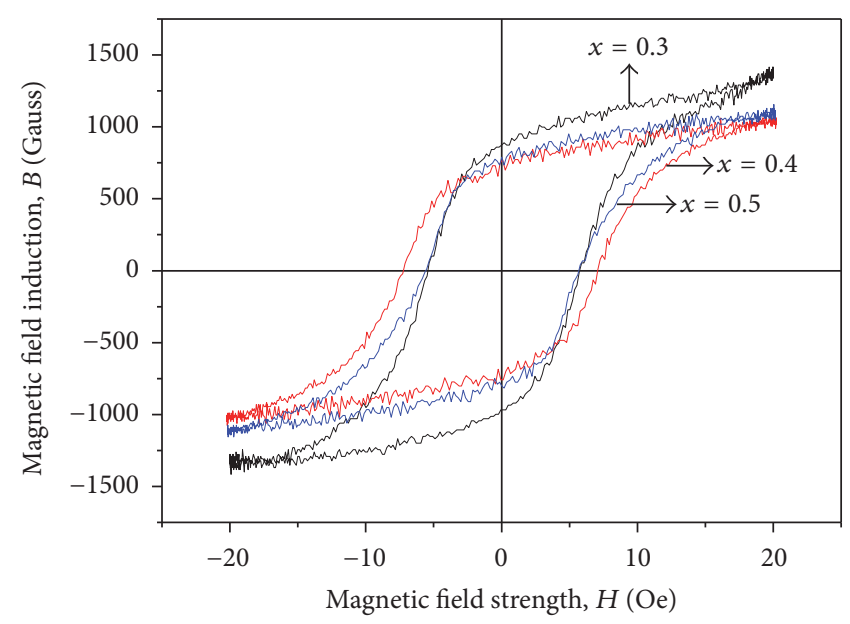

(b)

Figure 9: $B$ - $H$ loops of microwave sintered $\mathrm{Ni}_{0.8-x} \mathrm{Cu}_{x} \mathrm{Zn}_{0.2} \mathrm{Fe}_{2} \mathrm{O}_{4}$ ferrites for (a) $x=0.0, x=0.1$, and $x=0.2$ and (b) $x=0.3, x=0.4$, and $x=0.5$.

As the drive frequency increases and matches the precision frequency, the precessing spins absorb power from the drive field and as a result $\mu_{i}^{\prime}$ decreases. It is therefore expected that $\mu_{i}^{\prime}$ will begin to decrease at a lower frequency if $\mu_{i}^{\prime}$ is large. This is in agreement with the experimentally observed values. This means that the only loss mechanism in ferrites is the spin resonance [26]. It has also been reported that the decreasing trend in permeability with increasing frequency is due to nonmagnetic impurities between grains and intragranular pores which act as pinning points. This considerably reduces the contribution of the motion of spin and domain walls to the permeability [12].

Two-Magnetizing Phenomenon. Spin rotation and domain wall motion accounting for the permeability of polycrystalline ferrite can be represented as $\mu_{i}=1+\chi_{\omega}+\chi_{\text {spin }}$, where $\chi_{\omega}=3 \pi M_{s}^{2} D / 4 v$, and $\chi_{\text {spin }}=2 \pi M_{s}^{2} / K_{u}$, where $\chi_{\omega}$ is susceptibility due to domain wall motion, $\chi_{\text {spin }}$ is susceptibility due to spin rotation, $M_{s}$ is saturation magnetization, $D$ is average grain diameter, $v$ is domain wall energy, and $K_{u}$ is total anisotropy constant [27]. Accordingly, $\mu_{i}^{\prime}$ varies linearly with grain size. Globus et al. [28] also assumed that permeability arising due to wall motion is likely to vary linearly with grain size while permeability contribution to spin rotation was assumed to be independent of grain size. Globus [29] found linear dependence of permeability on grain size for several $\mathrm{NiZn}$ ferrites. These reasons account for the abrupt increase in initial permeability with increasing $\mathrm{Cu}^{2+}$ content, which attains a maximum value at $0.3 \mathrm{Cu}^{2+}$ concentration (as shown in Table 2). The higher value of grain size possibly explains the higher value of initial permeability for the microwave sintered specimens than the conventionally sintered ones.

3.6. Saturation Magnetization $\left(M_{s}\right)$. B-H loops of various compositions of $\mathrm{Ni}_{0.8-x} \mathrm{Cu}_{x} \mathrm{Zn}_{0.2} \mathrm{Fe}_{2} \mathrm{O}_{4}$ ferrites are depicted in Figures 9 and 10. The variation of $4 \pi M_{s}$ values with
$\mathrm{Cu}^{2+}$ ion content is shown in Table 2. These values are low as compared to those reported by Dimri et al. [14]. The exchange interaction between the ions at the tetrahedral (A) sites and octahedral (B) sites in the crystallographic lattice is responsible for the variation of saturation magnetization $M_{s}$ with concentration of $\mathrm{Cu}^{2+}$ ions. In spinel ferrites, $\mathrm{Zn}^{2+}$ ions occupy the A site while $\mathrm{Ni}^{2+}$ and $\mathrm{Cu}^{2+}$ ions have strong preference for the $\mathrm{B}$ site and $\mathrm{Fe}^{3+}$ ions partially occupy the $\mathrm{A}$ and $\mathrm{B}$ sites. $\mathrm{Fe}^{3+}\left(\mathrm{d}^{5}\right), \mathrm{Ni}^{2+}\left(\mathrm{d}^{8}\right), \mathrm{Cu}^{2+}\left(\mathrm{d}^{9}\right)$, and $\mathrm{Zn}^{2+}$ $\left(d^{10}\right)$ cations of the ferrite have $5,2,1$, and 0 unpaired electrons, respectively, and some magnetic moment in $\mu_{\mathrm{B}}$ [1]. The resultant magnetization, $M$, is the difference of the magnetizations $M_{\mathrm{B}}$ and $M_{\mathrm{A}}$ of the $\mathrm{B}$ and A sublattices, respectively, whereby the former generally has a higher value. As the magnetic moment of $\mathrm{Cu}^{2+}\left(1.3 \mu_{\mathrm{B}}\right)$ is less than that of $\mathrm{Ni}^{2+}\left(2.3 \mu_{\mathrm{B}}\right)$, the substitution of $\mathrm{Ni}^{2+}$ with $\mathrm{Cu}^{2+}$ will decrease the net magnetic moment and exchange interaction between $\mathrm{A}$ and $\mathrm{B}$ sublattices [30]. Consequently, $4 \pi M_{s}$ values will decrease. But the experimental values of $4 \pi M_{s}$ are found to increase with $\mathrm{Cu}^{2+}$ ion concentration up to $x=0.3$. This is indicative of the presence of $\mathrm{Cu}^{2+}$ ions on the $\mathrm{A}$ sites. Rahman and Ahmed [7] have also reported the presence of $\mathrm{Cu}^{2+}$ ions on the $\mathrm{A}$ sites. Hence, $\mathrm{Cu}^{2+}$ ions may have a tendency to occupy both sublattices [31]. This can alter the cation distribution to a greater extent which is a deciding factor in determining the overall magnetic properties. The incorporation of $\mathrm{Cu}^{2+}$ ions will force some of the $\mathrm{Fe}^{3+}$ ions to migrate from the A sites to the $\mathrm{B}$ sites. With the magnetic moment of $\mathrm{Fe}^{3+}$ ions $\left(5 \mu_{\mathrm{B}}\right)$ being greater than that of $\mathrm{Cu}^{2+}$ ions, the migration causes an increase in magnetic moment of $\mathrm{B}$ sites and a decrease in magnetic moment of A sites. This results in an increase in net magnetization $M=M_{\mathrm{B}}-M_{\mathrm{A}}$. With further addition of $\mathrm{Cu}$ ions, deviations are observed. More $\mathrm{Fe}^{3+}$ ions migrate to the $\mathrm{B}$ sites thereby increasing the magnetic moment of the $B$ site. The magnetic moments of the remaining few $\mathrm{Fe}^{3+}$ ions on the $\mathrm{A}$ sites are no longer able to align all the moments of the $\mathrm{B}$ ions antiparallel to themselves, 


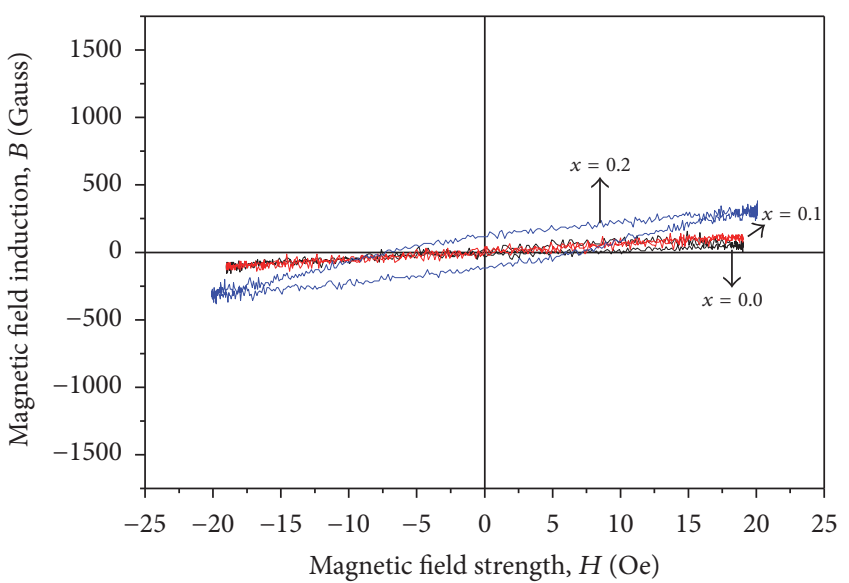

(a)

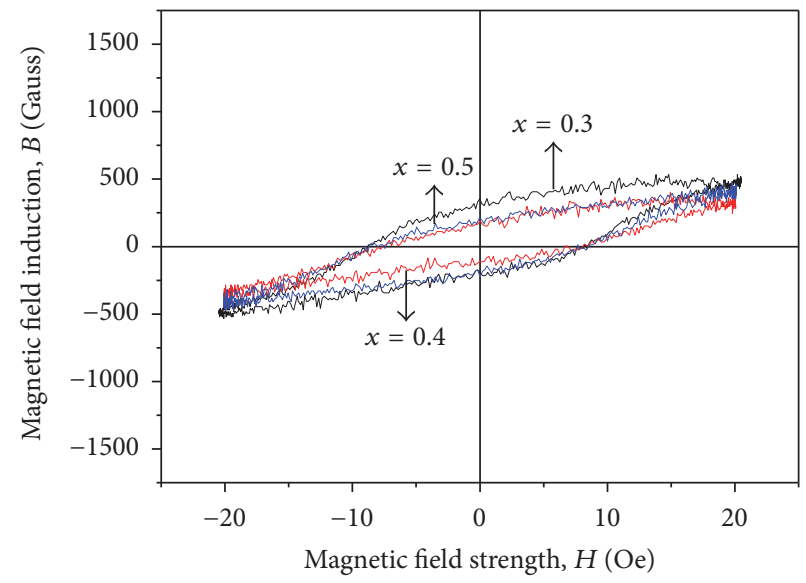

(b)

FiguRE 10: $B$ - $H$ loops of conventionally sintered $\mathrm{Ni}_{0.8-x} \mathrm{Cu}_{x} \mathrm{Zn}_{0.2} \mathrm{Fe}_{2} \mathrm{O}_{4}$ ferrites for (a) $x=0.0, x=0.1$, and $x=0.2$ and (b) $x=0.3, x=0.4$, and $x=0.5$.

TABLE 3: Hysteresis parameters of $\mathrm{Ni}_{0.8-x} \mathrm{Cu}_{x} \mathrm{Zn}_{0.2} \mathrm{Fe}_{2} \mathrm{O}_{4}$ ferrites.

\begin{tabular}{lcccc}
\hline \multirow{2}{*}{ Samples “ $x$ ” } & \multicolumn{2}{c}{ Microwave sintering } & \multicolumn{2}{c}{ Conventional sintering } \\
& $B_{r}$ (Gauss) & $B_{r} / B_{m}$ & $B_{r}$ (Gauss) & $B_{r} / B_{m}$ \\
\hline 0.0 & 23 & 0.160 & 21 & 0.135 \\
0.1 & 72 & 0.251 & 19 & 0.136 \\
0.2 & 712 & 0.630 & 118 & 0.309 \\
0.3 & 932 & 0.659 & 244 & 0.458 \\
0.4 & 729 & 0.678 & 149 & 0.370 \\
0.5 & 772 & 0.668 & 181 & 0.382 \\
\hline
\end{tabular}

as this is opposed by the negative B-B exchange interaction, which remains unaffected [31]. Therefore, a decrease in net magnetization is observed.

Apart from the preferential occupancy of particular lattice sites, the compositional variation of $M_{s}$ may be affected by the microstructure. Higher magnetization value is found for bigger grain size. Hence, it is observed that there is enhancement of $4 \pi M_{s}$ up to $\mathrm{Cu}^{2+}$ ion concentration of 0.3 .

Higher magnetization values $4 \pi M_{s}$ for the microwave sintered samples may be due to the alteration of charge distributions inside the magnetic lattice by the surrounding electromagnetic field and their contribution to the uncoupling effect. The uncoupling effect between the charged ions necessitates a high magnetic field to align them in the direction of the applied field [5].

Lower coercivity values were exhibited by the microwave sintered samples. This can be a result of larger grains obtained in microwave sintered samples. The number of domain walls is higher in larger grains and, with the increase in the number of walls, the contribution to the magnetization or demagnetization due to wall movement versus due to domain rotation increases. Hence, samples with bigger grains are expected to have low coercivity [32].

The squareness of the hysteresis loop is characterized by the remanence ratio $B_{r} / B_{m}$ [32] (Table 3 ). The greater the value of $B_{r} / B_{m}$, the greater the squareness of the loop and the greater the potential for application in memory devices. This ratio increases with the increase in concentration of the $\mathrm{Cu}$ ions and is maximum for the compositions $x=0.3$ and $x=0.4$ of the conventional and microwave sintered samples, respectively.

\section{Conclusions}

Nanoparticles of $\mathrm{NiCuZn}$ ferrite with compositional formula $\mathrm{Ni}_{0.8-x} \mathrm{Cu}_{x} \mathrm{Zn}_{0.2} \mathrm{Fe}_{2} \mathrm{O}_{4}$ (where $0.0 \leq x \leq 0.5$ ) were successfully synthesized by citrate precursor method. Microwave and conventional sintering techniques have been adopted. The low temperature sintering enabled the ferrites to possess good electromagnetic properties which will make them suitable for MLCI applications. Low value of dielectric constant is one of such features which is desirable for high frequency applications. The XRD results showed that the nanoparticles have high phase purity, crystallinity, and spinel ferrite nature. The grain size is found to increase with $\mathrm{Cu}^{2+}$ ion incorporation up to $\mathrm{Cu}^{2+}$ content of 0.3 . Densification is observed with $\mathrm{Cu}^{2+}$ addition. The $\mathrm{Cu}^{2+}$ content has a significant effect on the magnetic and dielectric properties. The dielectric constant increased for all the compositions while magnetization increased and then decreased with increasing $\mathrm{Cu}^{2+}$ concentration. Now, looking from the perspective of sintering mechanism, microwave sintered pellets are found to contain relatively larger grains compared with the corresponding conventionally sintered samples, thus indicating an enhancement effect. Dielectric measurements indicated low dielectric constant values for the microwave sintered samples compared to the corresponding specimens sintered by the conventional technique, which are generally preferable for high frequency applications. Magnetic property measurements showed that $\mathrm{Ni}_{0.8-x} \mathrm{Cu}_{x} \mathrm{Zn}_{0.2} \mathrm{Fe}_{2} \mathrm{O}_{4}$ sintered by both microwave and conventional techniques exhibited the soft ferrite behaviour with high magnetization and low coercivity for the microwave sintered specimens. 


\section{Conflicts of Interest}

The authors declare that there are no conflicts of interest regarding the publication of this paper.

\section{Acknowledgments}

The authors are grateful to (i) Dr. Raghumani Ningthoujam (BARC, Mumbai) for the XRD of the microwave sintered samples and (ii) Manipur University for the SEM measurements.

\section{References}

[1] H. Harzali, F. Saida, A. Marzouki et al., "Structural and magnetic properties of nano-sized NiCuZn ferrites synthesized by coprecipitation method with ultrasound irradiation," Journal of Magnetism and Magnetic Materials, vol. 419, pp. 50-56, 2016.

[2] B. S. Tewari, A. Dhyani, S. K. Joshi, S. Dubey, and K. Pandey, "Study of magnetic property of Sn doped Ni-Zn-Fe nanoparticles," Conference Papers in Science, vol. 2014, Article ID 816970, 4 pages, 2014.

[3] S. Sharma, K. Verma, U. Chaubey, V. Singh, and B. R. Mehta, "Influence of $\mathrm{Zn}$ substitution on structural, microstructural and dielectric properties of nanocrystalline nickel ferrites," Materials Science and Engineering B, vol. 167, no. 3, pp. 187-192, 2010.

[4] L. Zhang and Y. Wu, "Sol-gel synthesized magnetic $\mathrm{MnFe}_{2} \mathrm{O}_{4}$ spinel ferrite nanoparticles as novel catalyst for oxidative degradation of methyl orange," Journal of Nanomaterials, vol. 2013, Article ID 640940, 6 pages, 2013.

[5] P. Yadoji, R. Peelamedu, D. Agrawal, and R. Roy, "Microwave sintering of $\mathrm{Ni}-\mathrm{Zn}$ ferrites: comparison with conventional sintering," Materials Science and Engineering B, vol. 98, no. 3, pp. 269-278, 2003.

[6] W.-C. Hsu, S. C. Chen, P. C. Kuo, C. T. Lie, and W. S. Tsai, "Preparation of NiCuZn ferrite nanoparticles from chemical co-precipitation method and the magnetic properties after sintering," Materials Science and Engineering B, vol. 111, no. 23, pp. 142-149, 2004.

[7] I. Z. Rahman and T. T. Ahmed, "A study on Cu substituted chemically processed Ni-Zn-Cu ferrites," Journal of Magnetism and Magnetic Materials, vol. 290-291, pp. 1576-1579, 2005.

[8] A. Verma, T. C. Goel, and R. G. Mendiratta, "Frequency variation of initial permeability of NiZn ferrites prepared by the citrate precursor method," Journal of Magnetism and Magnetic Materials, vol. 210, no. 1-3, pp. 274-278, 2000.

[9] S. Das, A. K. Mukhopadhyay, S. Datta, and D. Basu, "Prospects of microwave processing: an overview," Bulletin of Materials Science, vol. 32, no. 1, pp. 1-13, 2009.

[10] K. M. Batoo and M. S. Ansari, "Low temperature-fired Ni$\mathrm{Cu}-\mathrm{Zn}$ ferrite nanoparticles through auto-combustion method for multilayer chip inductor applications," Nanoscale Research Letters, vol. 7, article 112, 14 pages, 2012.

[11] J. J. Shrotri, S. D. Kulkarni, C. E. Deshpande et al., "Effect of $\mathrm{Cu}$ substitution on the magnetic and electrical properties of $\mathrm{Ni}$-Zn ferrite synthesized by soft chemical method," Materials Chemistry and Physics, vol. 59, no. 1, pp. 1-5, 1999.

[12] A. K. M. Akther Hossain and M. L. Rahman, "Enhancement of microstructure and initial permeability due to $\mathrm{Cu}$ substitution in $\mathrm{Ni}_{0.50-x} \mathrm{Cu}_{x} \mathrm{Zn}_{0.50} \mathrm{Fe}_{2} \mathrm{O}_{4}$ ferrites," Journal of Magnetism and Magnetic Materials, vol. 323, no. 15, pp. 1954-1962, 2011.

[13] I. Soibam, S. Phanjoubam, and C. Prakash, "Magnetic and Mössbauer studies of Ni substituted Li-Zn ferrite," Journal of Magnetism and Magnetic Materials, vol. 321, no. 18, pp. 27792782, 2009.

[14] M. C. Dimri, A. Verma, S. C. Kashyap, D. C. Dube, O. P. Thakur, and C. Prakash, "Structural, dielectric and magnetic properties of NiCuZn ferrite grown by citrate precursor method," Materials Science and Engineering B, vol. 133, no. 1-3, pp. 42-48, 2006.

[15] M. M. Haque, M. Huq, and M. A. Hakim, "Densification, magnetic and dielectric behaviour of $\mathrm{Cu}$-substituted $\mathrm{Mg}-\mathrm{Zn}$ ferrites," Materials Chemistry and Physics, vol. 112, no. 2, pp. 580-586, 2008.

[16] B. Thangjam and I. Soibam, "FT-IR study of Cu substituted Ni$\mathrm{Zn}$ ferrites prepared by citrate precursor method," Advanced Materials Proceedings, vol. 2, no. 3, pp. 205-208, 2017.

[17] F. Alam, M. H. R. Khan, H. N. Das, and A. K. M. Akhter, "Structural and magnetic properties of $\mathrm{Mn}_{0.50-x} \mathrm{Zn}_{0.50} \mathrm{Cu}_{x} \mathrm{Fe}_{2} \mathrm{O}_{4}$," Materials Sciences and Applications, vol. 4, pp. 831-838, 2013.

[18] M. Yan and J. Hu, "Microwave sintering of high-permeability $\left(\mathrm{Ni}_{0.20} \mathrm{Zn}_{0.60} \mathrm{Cu}_{0.20}\right) \mathrm{Fe}_{1.98} \mathrm{O}_{4}$ ferrite at low sintering temperatures," Journal of Magnetism and Magnetic Materials, vol. 305, no. 1, pp. 171-176, 2006.

[19] S. Choudhury, M. A. Bhuiyan, and S. M. Hoque, "Effect of sintering temperature on apparent density and transport properties of $\mathrm{NiFe}_{2} \mathrm{O}_{4}$ synthesized from nanosize powder of $\mathrm{NiO}$ and $\mathrm{Fe}_{2} \mathrm{O}_{3}$," International Nano Letters, vol. 1, no. 2, pp. 111116, 2011.

[20] I. Soibam, S. Phanjoubam, H. B. Sharma, H. N. K. Sarma, R. Laishram, and C. Prakash, "Effects of Cobalt substitution on the dielectric properties of Li-Zn ferrites," Solid State Communications, vol. 148, no. 9-10, pp. 399-402, 2008.

[21] T. Jahanbin, M. Hashim, and K. Amin Mantori, "Comparative studies on the structure and electromagnetic properties of $\mathrm{Ni}-\mathrm{Zn}$ ferrites prepared via co-precipitation and conventional ceramic processing routes," Journal of Magnetism and Magnetic Materials, vol. 322, no. 18, pp. 2684-2689, 2010.

[22] A. Thakur and M. Singh, "Preparation and characterization of nanosize $\mathrm{Mn}_{0.4} \mathrm{Zn}_{0.6} \mathrm{Fe}_{2} \mathrm{O}_{4}$ ferrite by citrate precursor method," Ceramics International, vol. 29, no. 5, pp. 505-511, 2003.

[23] M. A. Rahman, M. A. Rahman, and A. K. M. A. Hossain, "Effect of $\mathrm{Cu}^{2+}$ substitution on structural, magnetic and transport properties of $\mathrm{Fe}_{2.5} \mathrm{Zn}_{0.5-x} \mathrm{Cu}_{x} \mathrm{O}_{4}$," Journal of Magnetism and Magnetic Materials, vol. 369, pp. 168-175, 2014.

[24] Z. Yue, J. Zhou, L. Li, X. Wang, and Z. Gui, "Effect of copper on the electromagnetic properties of $\mathrm{Mg}-\mathrm{Zn}-\mathrm{Cu}$ ferrites prepared by sol-gel auto-combustion method," Materials Science and Engineering B, vol. 86, no. 1, pp. 64-69, 2001.

[25] P. B. Belavi, G. N. Chavan, L. R. Naik, R. Somashekar, and R. K. Kotnala, "Structural, electrical and magnetic properties of cadmium substituted nickel-copper ferrites," Materials Chemistry and Physics, vol. 132, no. 1, pp. 138-144, 2012.

[26] B. D. Cullity and C. D. Graham, Introduction to Magnetic Materials, John Wiley \& Sons, Hoboken, NJ, USA, 2009.

[27] J. Hu and M. Yan, "Preparation of high-permeability NiCuZn ferrite," Journal of Zhejiang University SCIENCE B, vol. 6, no. 6, pp. 580-583, 2005.

[28] A. Globus, P. Duplex, and M. Guyot, "Determination of initial magnetization curve from crystallites size and effective anisotropy field," IEEE Transactions on Magnetics, vol. 7, no. 3, pp. 617-622, 1971. 
[29] A. Globus, "Magnetization mechanisms some physical considerations about the domain wall size theory of magnetization mechanisms," Journal de Physique Colloques, vol. 38, no. 1, pp. C1-1-C1-5, 1977.

[30] H. Su, H. Zhang, X. Tang, and X. Xiang, "High-permeability and high-Curie temperature NiCuZn ferrite," Journal of Magnetism and Magnetic Materials, vol. 283, no. 2-3, pp. 157-163, 2004.

[31] J. Smit and H. P. J. Wijn, Ferrites, Philips, Technical Library, Eindhovan, The Netherlands, 1959.

[32] A. Verma, T. C. Goel, R. G. Mendiratta, and P. Kishan, "Magnetic properties of nickel-zinc ferrites prepared by the citrate precursor method," Journal of Magnetism and Magnetic Materials, vol. 208, no. 1, pp. 13-19, 2000. 

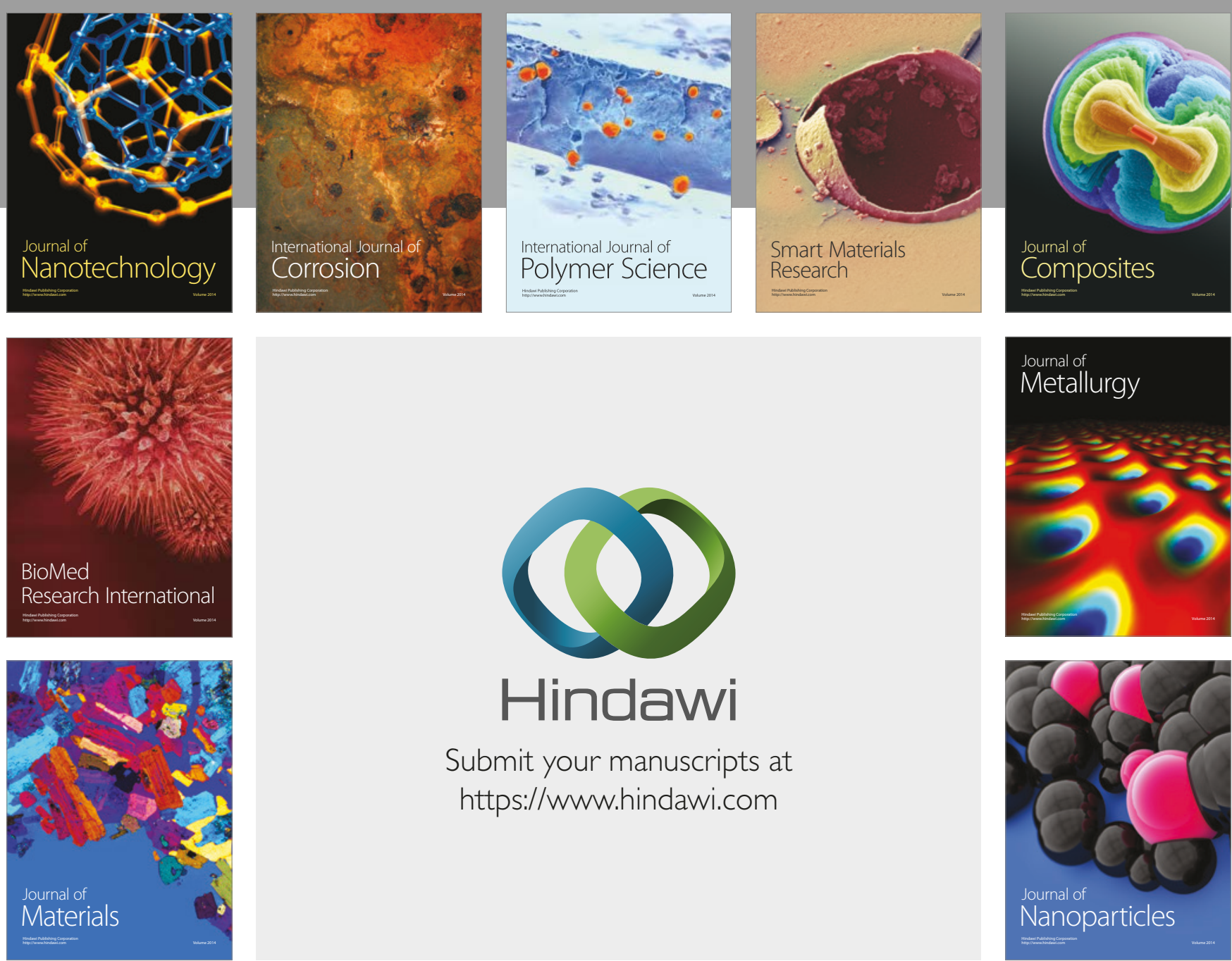

\section{Hindawi}

Submit your manuscripts at

https://www.hindawi.com
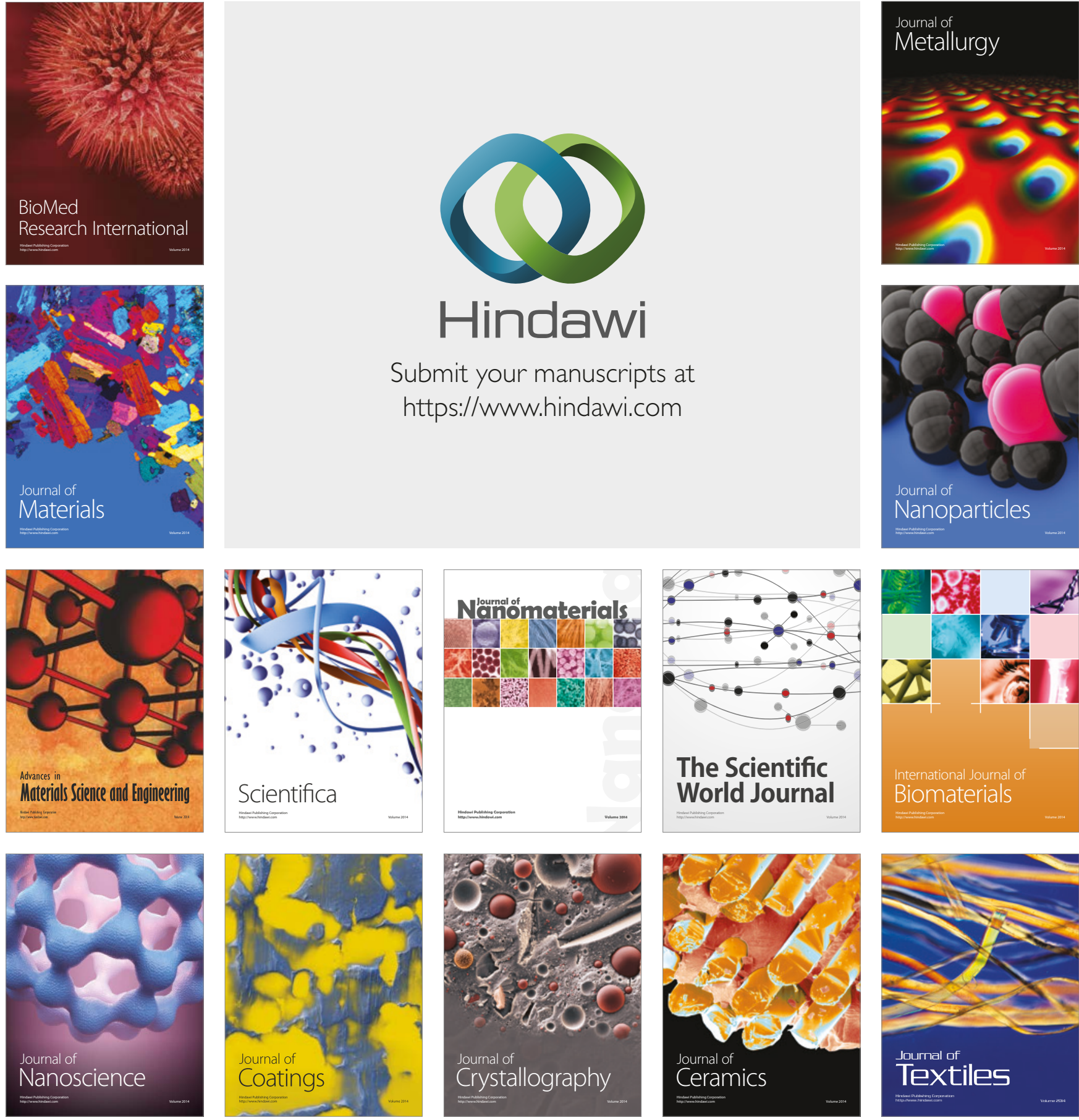

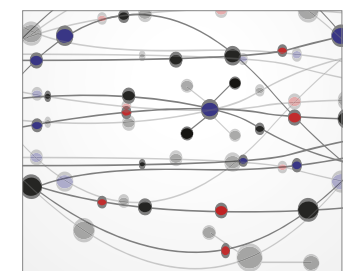

The Scientific World Journal
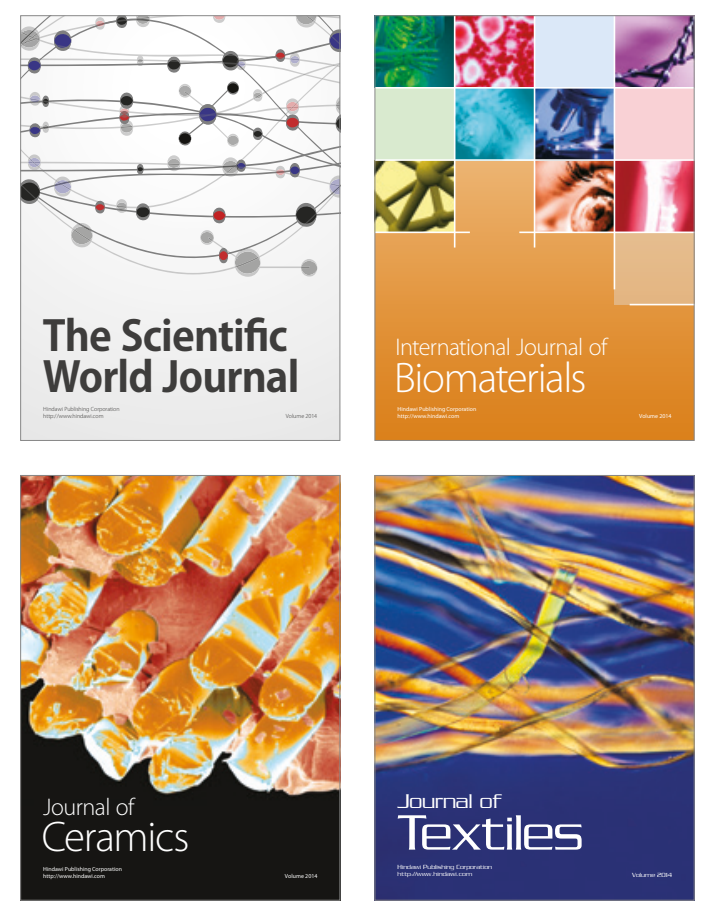